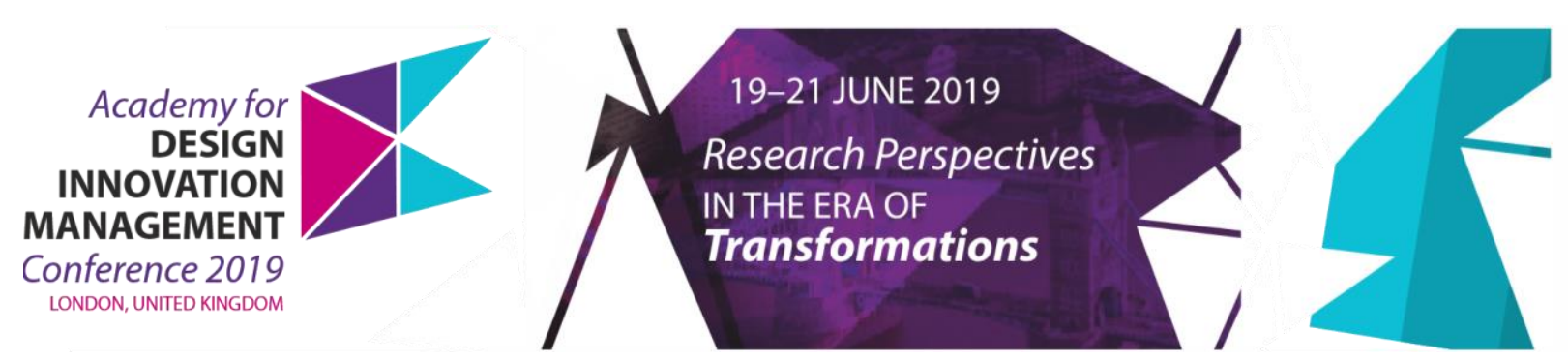

\title{
Design for Meaning of Smart Connected Products
}

\author{
VITALI Ilariaa; ARQUILLA Venanzio ${ }^{a}$ and RIFINO Innocenzo ${ }^{b}$ \\ a Politecnico di Milano, Italy \\ b Studio Habits, Italy \\ * corresponding author e-mail: ilaria.vitali@polimi.it \\ doi: 10.33114/adim.2019.03.251
}

\begin{abstract}
This paper tackles the topic of meaning-driven innovation from a Product Design perspective. It focuses on the design of Smart Connected Products: internet-enabled phygital products that blend hardware and software. This category of products represents a positive field of exploration for meaning-driven innovation. The paper highlights three kinds of meaning that are relevant for Smart Connected Products: the meaningful identity of the object as product category, the meaning of the product in relation to its shape and functionality, and in relation to a phygital ecosystem. The paper reflects on the methods that can support designers in the development of meaningful smart products and presents the "Mapping the loT" Toolkit, a downloadable tool that guides in specific activities aimed at framing the product's meaning. Tests with the Toolkit proved the effectiveness of using cards with critical questions as a way to deepen design concepts and reach a common, meaningful vision.
\end{abstract}

Keywords: Meaning-driven innovation, Product Design, Smart Connected Products, IoT, Design Tool

\section{Types of innovation and meaning driven innovation}

There are two main kinds of innovation that concerns products: incremental and radical.

Run-of-the-mill, incremental innovations represent a progressive improvement of certain parameters of the product. Norman and Verganti (2014) compare it to a "hill climbing" activity, since it tries to modify iteratively few product's characteristics to achieve newer, better versions, and gain more quality over time. It is not known in advance where is the peak of this hill to be climbed. The techniques of Human Centered Design, with fast iterative cycles of research, prototyping, and user testing, are proved to facilitate in the path towards incremental innovation.

Radical innovation instead, aims not to climb the same hill, but to find a new one with a higher potential. This kind of innovation has been labeled in different ways such as radical, discontinuous, generational or breakthrough innovation. Interest in radical innovation originated with Schumpeter (1934) and was seen as a way to challenge the power of monopolists.

Dahlin and Behrens (2005) defined three conditions to allow ex-ante identification of potential radical innovation and ex post evaluation of its impact. To be defined as radical, an invention must be novel and unique, hence dissimilar from prior inventions, and finally, it must be adopted and influence the content of future inventions: this transforms it in a successful change agent for the industry.

This work is licensed under a Creative Commons Attribution-NonCommercialShare Alike 4.0 International License. https://creativecommons.org/licenses/by-nc-sa/4.0/ 
Radical innovation can be driven by technological epiphanies, that open up to new possibilities and promote different visions, and by deliberate changes in the meaning of product (Verganti, 2017; Norman \& Verganti, 2014).

For example, when unspoken dynamics in sociocultural models arise, new meanings can re-align the industry to people's aspirations. This may be achieved by addressing new performance needs with attributes that are currently not valued by existing customers (Bowler \& Christensen 1995): a cited example is the Nintendo Wii, that radically innovated the market of gaming consoles because it focused on immersivity instead of on realistic graphic quality, the performance that was more valued by previous consoles.

For Norman \& Verganti (2014), radical innovation is seen as comparable to technology-push innovation: to reach it, it is crucial to develop a strong internal vision, rather than applying the iterative $\mathrm{HCl}$ methods.

In the next section the concepts of meaning and meaning driven innovation are deepened.

\section{Meaning and Meaning-driven Innovation}

In a simple way, it can be said that the "meaning" of a product is the "reason why" behind it, "the profound psychological and cultural reasons people use a product" (Verganti, 2011, p.384).

Going deeper into the nature of meaning, it has been widely accepted that is composed by a "bundle of components" that include both objective and subjective elements, such as experiences, feelings and images.

Meanings can be categorized in three types: lexical meaning, philosophical and psychological (Friedman and Lessig, 1986). Psycological meanings in particular are those focusing on a person's subjective perception and affective reactions (Szalay and Deese, 1978).

The meanings of a given object can be characterized by three dimensions: tangibility, emotionality and commonality. These dimensions have been discussed for example by Friedman (1986) and Hirschman (1980).

Tangibility concerns whether the attribute at the basis of meaning is tangible, in the sense that is objective and verifiable through the senses, instead of being subjective. It reflects if the meaning is located in the object itself or in the mind of the user.

The emotional side of product meaning is an important component of the meaning of an object, that cannot be detached from the objective side. Emotional responses to products can range from low to high intensity, going from simple affective reactions to true emotional experiences such as enjoyment and excitement.

The third dimension of meaning is that of commonality, that identifies the degree to which meaning is shared by members of the culture, or if it has a more individualized and personal character. It is linked to the source that is more responsible of the assignment of meaning to the object. For example, it is a cultural source if the meaning is assigned by advertising and shared media opinion. It is a personal source if meaning is created by the user through time, experience and interaction, such as an object received as a gift from a dear friend, your most comfortable pair of shoes, or your favourite toy when you were a child.

Different researchers focused on framing and categorizing diverse kind of meanings in order to deliver meaningful customer experiences. For example, Fournier (1991) defines eight categories of customer meanings: objects of utility, action, appreciation, transition, childhood, ritual enhancement, personaly identity, and position and role. While Diller et al. (2005) suggest fifteen categories of meaning: accomplishment, beauty, creation, community, duty, enlightenment, freedom, harmony, justice, oneness, redemption, security, truth, validation and wonder.

Product meanings have many connotations, depending on which theoretical perspective is hold, for example Affordance Theory, Product Semantics, Product Emotions. Thanks to the highly sociological nature of meanings, they can be shaped and in a sort of way controlled by designers (Giacomin, 2017).

This is a topic of study in the field of product semantics: while semiotics is the study of signs, semantics is the study of their meaning, and applied to design, it concerns how designers encode meaning into their products to communicate with the user.

Design is seen as a form of communication (Krippendorff \& Butter, 1984) in which the symbolic qualities of products are the messages to the user. The meaning of a product must be imagined connected from its contexts of use and in relation to the user's cognitive models (Krippendorff, 1989). 
Meanings can be attributed to artefacts through a process of semantization. Siefkes (2012) proposes seven principles of semantization: function, style, iconic associations, individual experiences, cultural allusions, connection to social groups, and connection to specific contexts.

Giacomin instead proposes a framework diagram of design for meaning that identifies if an artefact should adhere to an existing societial stereotype of if there is the possibility to define a new function, ritual or myth. He calls this "meaningfication" and defines it as

the use of data, ethnography, real fictions and co-creation for the purpose of designing artefacts based on new meanings that emerge from the interconnection of evolving patterns of technology, experience, personal identity, societal identity, value assignation and consumption.

The next section applies the topic of meaning to the design of smart products.

\section{Design for Meaning of Smart Connected Products}

In this paper the term "design" is used referring to the industrial design practice:

Design is a strategic-problem solving process able to drive innovation through innnovative products, systems, services, and experiences (World Design Organization, 2015)

Design is an activity of meaning creation. As said by Krippendorff, "Design is making sense of things".

The term "Smart Connected Products" identifies tangible products with computational capabilities, conneced to the Internet and - possibly - to other objects. Their characteristic feature is being cyber-physical (or "phygital"): it means having both a tangible/physical form and a digital/intangible part. This digital counterpart is what Greenfield (2006) calls "Informational shadow" and what Semmelhack (2013) defines as "Digital Avatar", and is one of the two states in wich the product exists.

Smart connected products are IoT products because they are part of the Internet of Things, "a world-wide network of interconnected objects uniquely addressable, based on standard communication protocols" (INFSO D4 \& RFID INFSO G.2, 2008), a network "Available anywhere, anytime, by anything and anyone (ITU, 2005).

This paper discusses why smart connected products are an eccellent field of application and exploration for meaning-driven innovation. The insights presented derive from the authors' academic and professional experience. In particular, the analysis of case studies of smart connected products (Vitali, 2015; Arquilla \& Vitali, 2016) led to the definition of a Toolkit to support designers and their teams in the development of loT products (Vitali, Rognoli \& Arquilla, 2016; Vitali \& Arquilla, 2018). The Toolkit is later described as a case study in this paper.

There are three kinds of meanings that are specifically relevant in the design of smart connected products.

- The meaningful identity of the object as product category

- The meaning of the product in relation to its shape and functionality

- The meaning of the product in relation to a phygital ecosystem

\section{The meaningful identity of the object as product category}

The suffix "Smart" is often abused to call attention to those products with relevant technological features, or that try to solve a specific problem in an intelligent way.

There is no univocal definition of what a "smart product" is (Gutierrez, et al, 2013), but the term is used to identify objects able to gather and process data (Kiritsis, 2011), with an autonomous and adaptive behavior (Maass and S. Janzen, 2007) providing a natural product-to-human interaction (Sabou et al, 2009) and active communication with users, environments or other products and systems (Venta, 2007).

Smart products suffer from identity crisis. They are not strongly culturally semantized. Their smartness is often translated in the ability of being controlled by a smartphone. This label of being "products with an app" links to meanings that don't communicate efficiently their worth and innovative elements: they are oftenseen as tech gadgets. What smart connected products need, is a well-designed identity and specific value proposition. "Focused, divergent and with a compelling tagline", like an effective blue ocean strategy (Kim and Mauborgne, 2005). 
Analyzing the consumer electronics market, three macro-categories of smart products emerge:

1. In the first case, the object is the technological, connected version of an analog product. For example, a smart water bottle that tracks how much water is drunk during the day, and that sets reminders for the user. Adding connectivity and technological features for this category of products can be seen as a radical innovation.

2. In the second case, the object is smart because it is the "smarter" version of an electronic/digital product. For example, an internet-connected weight scale that lets users record their weight in time. Adding connectivity for this category of products can be seen as an incremental innovation of its technological features.

3. In the third case, the object is smart because it combines new and existing functions, resulting in a new product category in the market. This kind of intervention tries to satisfy user needs in a radical new way. An example is the Roomba vacuum cleaner, that offers a new vision for home cleaning, combining initially unrelated topics of "home robots" and "vacuum cleaners".

The semantic dimension is a powerful source of competitive advantage (Battistella, Biotto, De Toni, 2012).

For all three categories, there is a need of semantization, or what Giacomin interprets as meaningfication: "customers are implicitly invited o think differently about the opportunities and value propositions which are on offer". (Giacomin, 2017). Innovative aspects of the products must be framed with new meanings.

In the first and second categories of smart products, there is also the need to clarify if the meanings of these smart products still align with the pre-existing meanings linked to their unconnected counterparts.

A successful example of this is represented by Nest, a connected thermostat, that through technology was able to shift the identity/meaning of the thermostat from a static, passive, and hard to personalize element of the house, towards an active and reactive element, that automatically answers to user's presence and habits. This shift from the pre-existing values led to a new vision for the market of thermostats.

Another example concerns the creation of new visions in the market of Smart TVs. Samsung reflects on the role in the room of large, wall-mounted TVs, trying to suggest new meanings. Samsung proposes "The Frame" a TV that when switched off becomes a personal piece of art displaying selected high-resolution images from a curated art gallery. In this way the TV acquires a meaning in the room, even when is not switched on. Another innovation is the "Samsung Ambient Mode", that lets the TV screen disappear, simulating the texture of the wall on which it is mounted. Complementarily, LG presented at CES 2019 "Signature R" a rollable OLED TV, that lets the screen physically appear only when needed.

In these examples the new meanings and visions redefine the architectural role of the TV, trying to anticipate future user whishes.
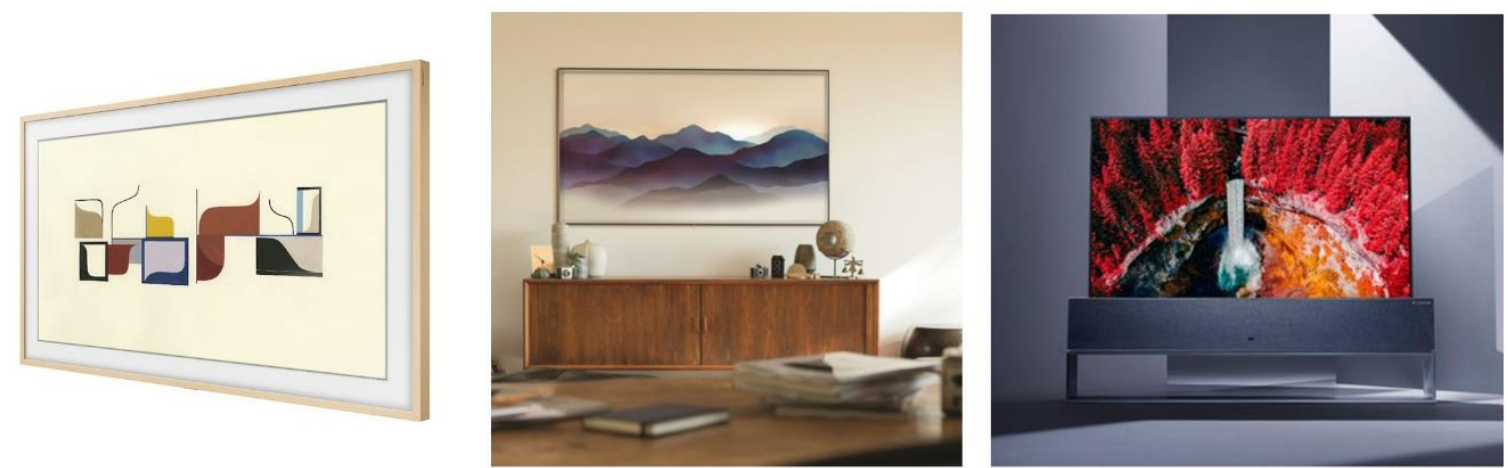

Figure 1: From left to right: Samsung "The Frame", Samsung "Ambient Mode", LG "Signature R"

The communication of the new values is crucial to redefines the smart product's identity and its position in the marketplace.

An exemplar case study of meaningful communication is the Apple Watch video series "Real Stories" (Apple, 2018, link in bibliography) that documents stories in which the functions of the smartwatch were able to deeply impact its users, for example by saving their life thanks to the heartbeat alarm notification, by calling an 
ambulance after a car accident, by calling for help for being rescued when lost at sea. With the interviews in the video, the smartwatch shows that it can be a meaningful device for health and safety, and not only a product aimed at sport athletes.

\section{The meaning of the product in relation to its shape and functionality}

The second level of meaning concerns the meaning in relation to the product's shape and functionality. This kind of meaning is traditionally addressed by the field of Product Semantics.

A short digression about analogic artifacts highlights what electronic products lack.

The shape of analogic artifacts is expressive. Through its affordance, the object "offers what it does because of what it is" (Gibson, 1977) and communicates how it could be used (Maier, \& Fadel, 2009), and the action through which interaction takes place (You \& Chen, 2007). Through shape and materials, the artifact communicates signs and messages that will be interpreted by the user in a subjective way. A successful design manages to communicate effectively and consistently to the receivers what the designer wanted to express. Therefore, the ability of the designer is to make the design comprehensible to the receiver/user by presenting qualities that will cause and fulfill certain expectations (Kazmierczak, 2003).

In electronic products, the link between shape and function is often lost. Electronic products are able to perform multiple functions which, however, are not evident, traceable or geometrically comprehensible in the shape of the artifact. Often this leads to anonymous shapes that do not communicate or invite interaction. Additionally, many smart products can't even be manipulated, since interaction takes place through external interfaces and via smartphone.

These aspects are the first three of what Krippendorff and Butter (1984) define as the four semantic infelicities in design: the product is undistinguishable or unidentifiable by the user, the product does not promote the correct way to manipulate it, it lacks transparency and it is not clear how to use it.

Therefore, a designer dealing with smart connected products should try to reduce these infelicities. One possible strategy is to design a shape that highlights and expresses the way of interaction with the product. Another strategy is to use metaphors to bring the meaning of the product closer to its primary function, and make it more comprehensible by aligning with the mental models of the user.

For example: what shape should a wireless router have? What is its archetype? Technically, a router is an antenna that transmits a signal, that is why many routers have visible antennas, for a better perceived performance. Instead, the wi-fi router "Asus Blue Cave" has a characteristic shape with a circular hole, marked by a blue finishing (Fig 2). This product doesn't show any antenna, and its shape is more similar to an air purifier. The shape uses the metaphor of "airflow" to relate to the flowing of data. In this way the shape is more meaningful, according to the product's function.
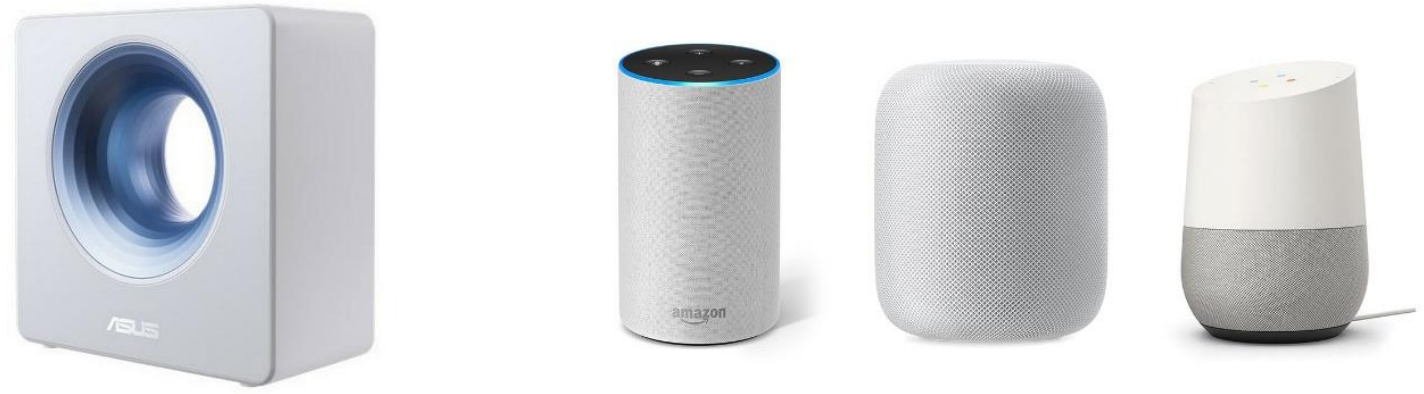

Figure 2: From left to right: Asus "Blue cave" wi-fi router and three smart speakers/home assistants. Amazon Echo $2^{\text {nd }}$ gen. with Alexa, Apple HomePod with Siri, Google Home with Google Assistant.

Following the same perspective, there is also the stimulating challenge to reflect on new metaphors and formal archetypes for emerging product categories such as the home assistants (Amazon Echo, Apple HomePod, Google home...), that currently materialize as cilindrical smart speakers. 


\section{The meaning of the product in relation to a phygital ecosystem}

Connected objects can be categorized according to their ability to process information, functionalities, and degree of interactivitiy. Rowland et al (2015) for example distinguish between multipurpose computer devices with powerful processing capabilities and rich interaction (e.g. smartphones, smart TV, game consoles ...), embedded devices specialized to answer to specific needs with less processing power (e.g. smart thermostat, smart weight scale, smart bottle ...), and connected sensors in which the gathered data is more important than the physical interaction with the product (e.g. flood sensor, air quality sensor ...).

Due to their phygital nature, connected products can be interacted with in a tangible and digital way. This blurs the line between product and service (Breschi et al, 2018). Analog products are "finished" once manufactured and sold, but connectivity allows the physical connected products to become avatars, physical stand-ins, for services.

Due to their dual nature, connected products follow more the dynamic logic of apps and software, rather than the static life of physical matter and hardware. "Design is never done" quotes Google, and so are loT products.

The connected product and its functions need to be framed in an ecosystem. The object is not isolated but social, and in relationship with physical and virtual things, data, and processes. The ability to position a smart product in a compatible ecosystem is crucial: ecosystems are "the competitive unit in the battle to establish leadership in loT" (Agrò, 2018). Objects can be augmented by services, or be included in the customer experience offered by a service provider. The more connections it has, the more the product could be considered as valueable and meaningful.

From the point of view of product design and UX design, this implies the need to design and manage a multiplicity of touchpoints, both physical and digital (Rowland et al, 2015). Therefore, when designing the product is important to define a balance between the tangible and digital aspects, in relation with its functions, and with the user's expectations. This will deeply impact the meaning of the artifact. For example, the choice of making a key feature of the product only accessible through an app rather than with physical interaction will create a different user experience. In many cases, such as for connected sensors, the object can't be interacted with directly and its meaning depends on its ability to provide a meaningful interaction with data.

At a more technical level and linked to the intangible, digital part of the product, few considerations need to be made regarding its ability to "work" even in the absence of an internet connection. The object may be internet-dependant to perform its primary function (e.g if it requires cloud connection to operate), or may perform part of its functions even in the absence of connection. This kind of technical choices can also have a great impact on the user experience.

The user experience of all these connected objects is simultaneously the Achilles' heel and the possible golden key for what makes loT devices like wearables meaningful to people (Adobe, 2017)

\section{Practical case study: designing meaningful connected products with the "Mapping the loT Toolkit"}

\section{Reflecting on the meaning of Smart connected products with the "Mapping the IoT" Toolkit}

Designing smart connected product is a complex task that requires effort from multidisciplinary teams. There are multiple aspects that designers need to be accounted for; in particular the design of the service offered by the product, the physical and digital interaction touchpoints, and the architecture of the product with its components.

The initial phase of the design process is crucial to frame the problem that the product is addressing, identifying possible user needs/desires, and envisioning new meanings, trying to go beyond superficial solutions, and to outline a coherent vision. This phase can also be called metadesign (Giaccardi \& Fischer, 2006). The aim of a metadesign process is to define the reason why of the project from the point of views of the user, of the market, and of the company itself. It is a phase of meaning creation.

The "Mapping the IoT" Toolkit (shortened as "MTIoT" Toolkit) aims at giving support during the design and metadesign process of smart connected products, helping multidisciplinary teams to discuss and work 
together, framing the problem, and creating a common vision. It is mainly intended for designers, but it can be used by whoever wants to approach the design of loT products. The Toolkit is distributed with a Creative Commons license and is free to download. The Toolkit helps designers and teams during 4 main phases of the design process, described by 4 activity guides. The activities supported by the guides are:

- "I want to start a new project" helps setting the research phase at the beginning of a new design project, identifying sources and selecting case studies

- "I want to analyze smart products" introduces a guided research exercise, exploring and comparing case studies

- "I want to brainstorm ideas" describes brainstorming exercises with the MTloT Deck

- "I want to make my concept better" offers an exercise to focus on the concept and its meaning

All activities exploit a set of cards - the "Mapping the loT" Deck - and may be used in conjunction with fillable canvases.

The cards of the deck and are divided in 7 different topics: user \& context, design, technology, interaction, experience, strategy, meaning. Cards are two sided and provide questions: the horizontal side of the card introduces a topic with one main question, while the vertical side deepens it with specific "what if" questions. Each topic is identified by a colour and a letter on the bottom of the card (Figure.3).

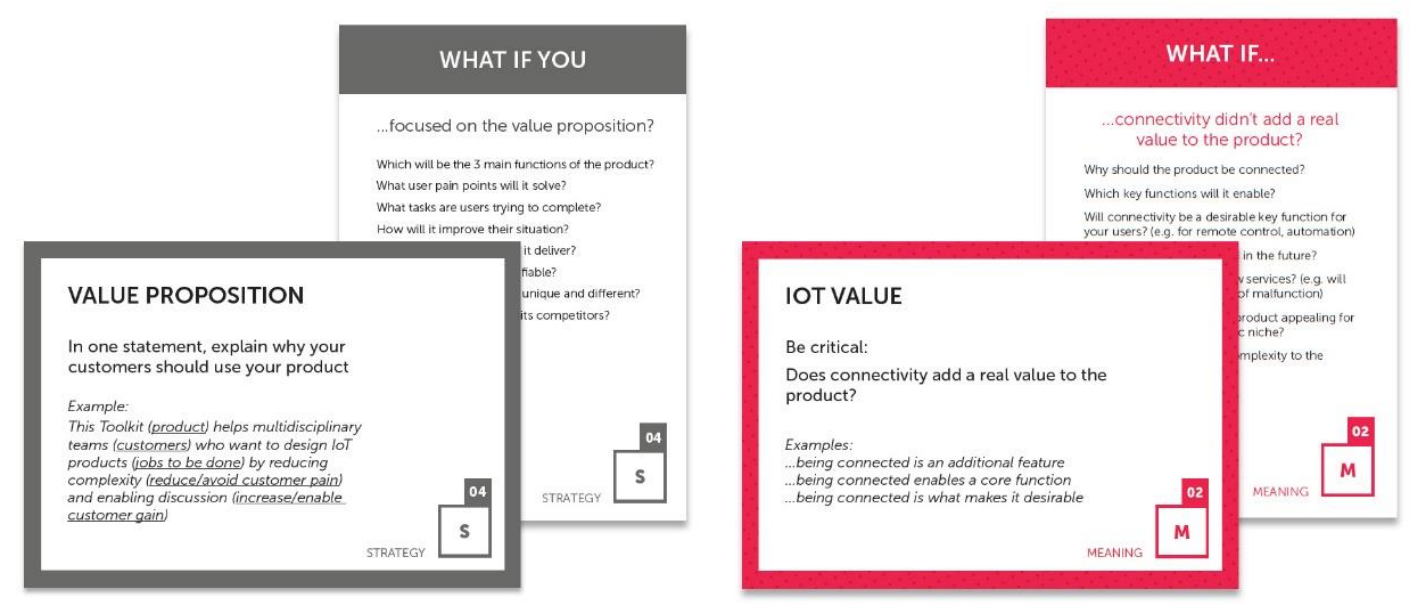

Figure 3: Example of a strategy card and of a meaning card of the "Mapping the loT" Deck

The cards are a knowledge repository to remember many of the features that need to be designed in complex smart products. They can be used as an input for reflection, and as diverging inspiration. The questions are an input to discuss the idea and the meaning of the product, therefore the cards have more impact at the beginning of the design process, between problem framing and concept definition. (Define, Develop, Deliver in Figure 4) (Vitali \& Arquilla, 2018). 


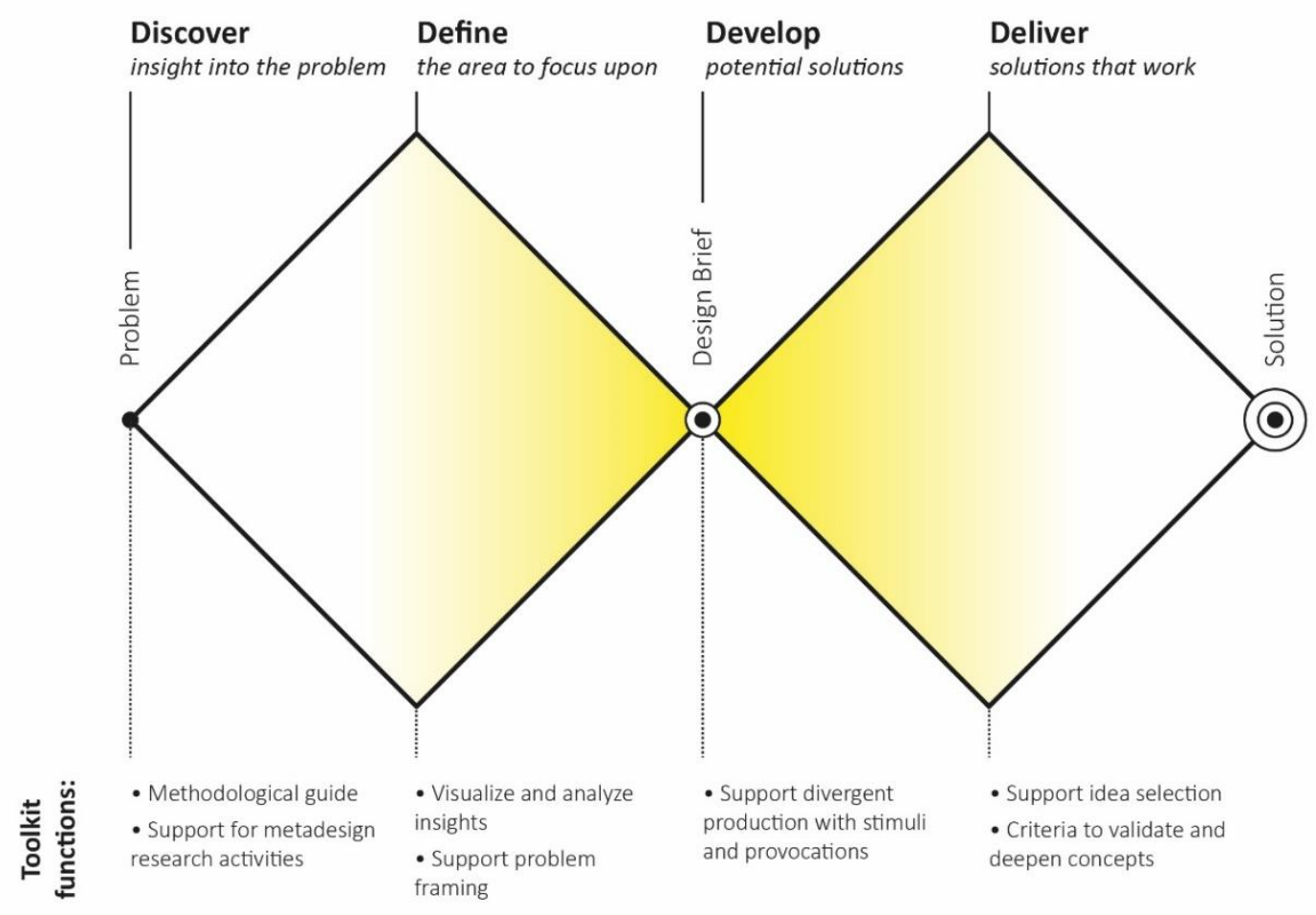

Figure 4: The "Mapping the loT" Toolkit functions positioned in relation to the double diamond representation of the design process.

The subdivision of the deck in topics pushes the team to concentrate on specific aspects of the product.

Group activities, facilitated by the Toolkit, are aimed at defining a clear vision for the smart product definition.

The elements of the MTIoT Toolkit that mostly focus on framing the meaning of the project are three: Meaning cards, Strategy cards, and the Activity guide "I want to make my concept better".

1. "Meaning" cards (the cards with fuchsia edges in Fig.3) propose critical questions that want to challenge the designer in observing the concept in an objective way, evaluating strengths and weaknesses.

2. "Strategy" cards (with grey edges in Fig.3) reflect on the uniqueness and market positioning of the concept, with a focus on its business model and competitor analysis. They want to support the development of the product's value proposition. These help to frame the meaning of the product for the market.

3. The Activity guide "I want to make my concept better" introduces an activity to be done in conjunction with the deck cards and the "sum up your smart-product idea" canvas (Fig.5). The canvas is split in 7 areas, that follow the cards'subdivision. It is printed and used to summarize the initial design concept of a smart product. Then the cards of the deck are used to discuss and deepen the initial idea with the team, by asking and answering questions. The exercises "forces" the designer to frame the idea and its meaning for the user/context, and for the market. 


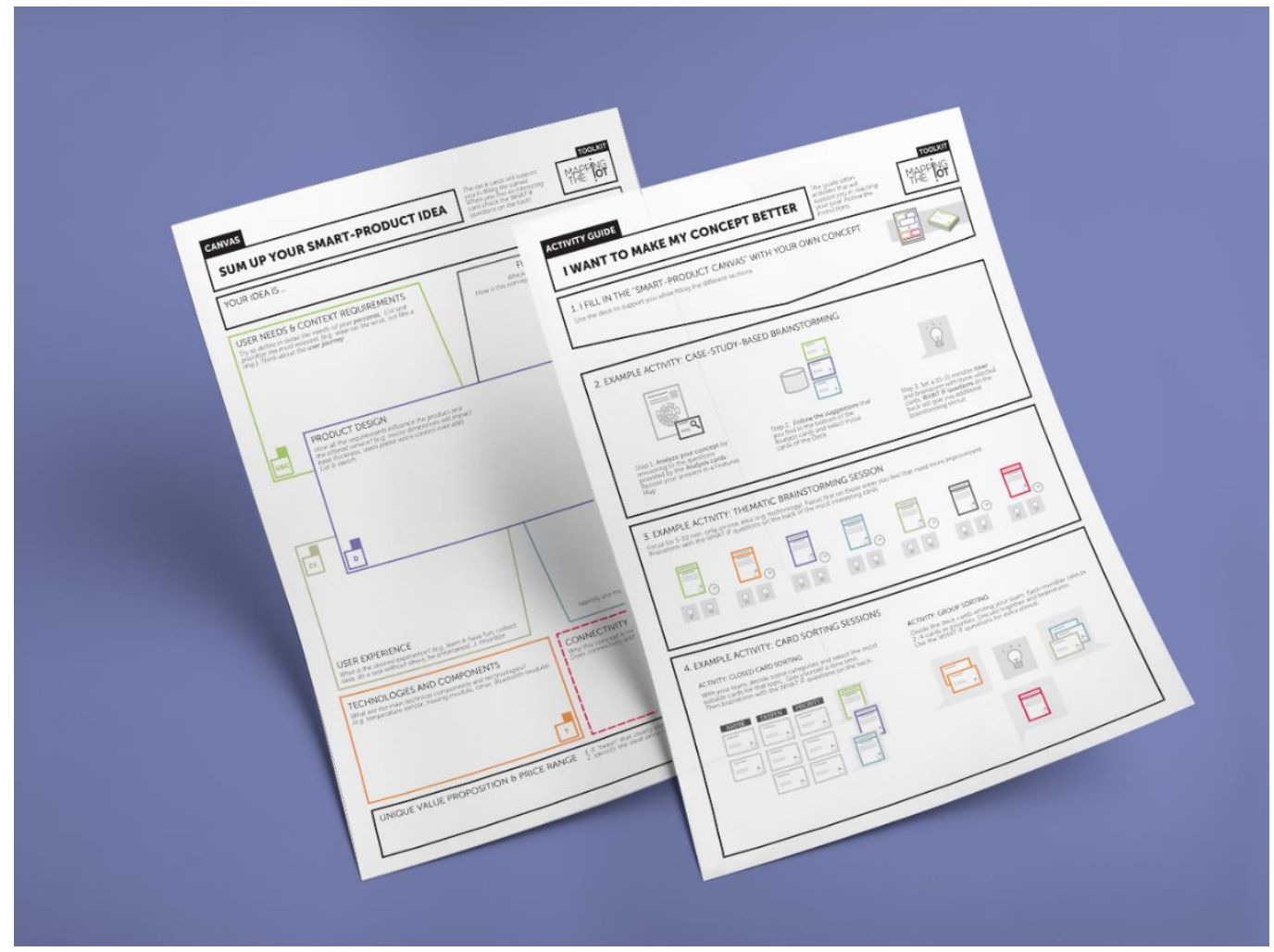

Figure 5: The Activity guide "I want to make my concept better" (on the right) describes an activity that uses the MTIoT deck cards and the "Sum up your smart-product idea" canvas (on the left)

The Toolkit has been tested and presented in different occasions, with academic peers, professionals and design students and is being formalized as a teaching support at Politecnico di Milano.

Among the recent tests and presentations:

- Academically: test at Miteinander at Chemnitz University of Technology 2018, DRS conference 2018, NordiCHI conference 2017

- Professionally: "Product of Things 2018" in Tel Aviv, "IoT Connected design event 2018" by loT Italy in Treviso, "Workshop Explore Innovation and design trends 2017" in Milan.

\section{Test $A$ and Test B following the "I want to make my concept better" Activity guide}

The MTIoT Toolkit is a kit that evolves constantily, following user insights: like smart connected products, it is upgradeable and is currently at its $4^{\text {th }}$ version. Recent structured tests focused specifically on the dimension of meaning generation.

This paper describes two Toolkit tests that involved students at their second year of Master's Degree in "Integrated Product Design" and in "Design and Engineering" at Politecnico di Milano Design School. In both cases students were designing smart products for their final course (Final Synthesis Studio) in collaboration with different companies (Braun, Midea, Enovia-Yape).

Test A involved students of the Master's course in Integrated Product Design. The aim of the course, called "Smart/Design Lab." was to develop innovative appliances that reflected on the topics of food, drinks, clothes care, and beyond-the-screen interaction.

27 groups of students tested their own initial idea by following the Toolkit activity "I want to make my concept better". They summarized their starting concept on the "sum-up your smart product idea" canvas, then used the cards to analyze and deepen their idea according to the areas proposed by the cards (user \& context, design, technology, interaction, experience, strategy, meaning).

Cards were used as a discussion tool inside the team, to identify the weaknesses and opportunities of their concept, to make choices and give it a stronger identity. The activity with the Toolkit was done without a 
facilitator, but the course staff was available for clarifications. The MTloT Toolkit was used like a cultural probe: groups could discuss freely, and they were asked to self-document their process. The day after the activity each group received a survey aimed to evaluate in a qualitative way what was the impact of the Toolkit on the project.

The limits of this test were:

- All groups were working on different projects, so the starting point for the use of the Toolkit wasn't exactly equal: some groups started with a more advanced concept than others.

- The activity in class lasted an afternoon, but the Toolkit was also used independently by some groups.

- The survey data were collected anonymously, unrelated to the individual group projects. Data are qualitative and represent the subjective point of view of the participants of the test.

Test B involved 32 students of the Master's course in "Design and Engineering". In this case all 9 groups of students started from the same brief: redesign an existing self-driving robot drone, by finding new use cases, meanings, identities.

The 9 groups of test B performed the same activity proposed to test A group: work with the team and summarize the starting idea in the "sum-up your smart product idea" canvas, then use the card to discuss together. In this case the activity lasted an afternoon, and at the end of the day the students received the survey.

The limits of this test concern the fact that the Toolkit was used in a more advanced moment of the design process. Few groups already decided some formal/functional elements of the product and were beginning the engineering process. In this test, the survey was administered individually and not filled as a group.

\section{Test results}

As mentioned before, the survey was anonymous and was aimed at probing the perceived impact of the Toolkit during the design of smart products. The survey employed closed and open questions. Closed statements were rated through a linkert scale ranging from 1 (strong disagree) to 5 (strong agree). In total, 60 surveys were collected.

The Mapping the IoT Toolkit received a positive response. In particular, the cards were appreciated, and many participants stated that they would use them again for their project. "Did you like the Toolkit approach" received an average response of 4.1 and "Would you use the cards again" an average of 4.0.

"The cards are a great medium; the questions are really well thought of and very helpful"

Evaluating the impact of the Mapping the loT Toolkit on the design project

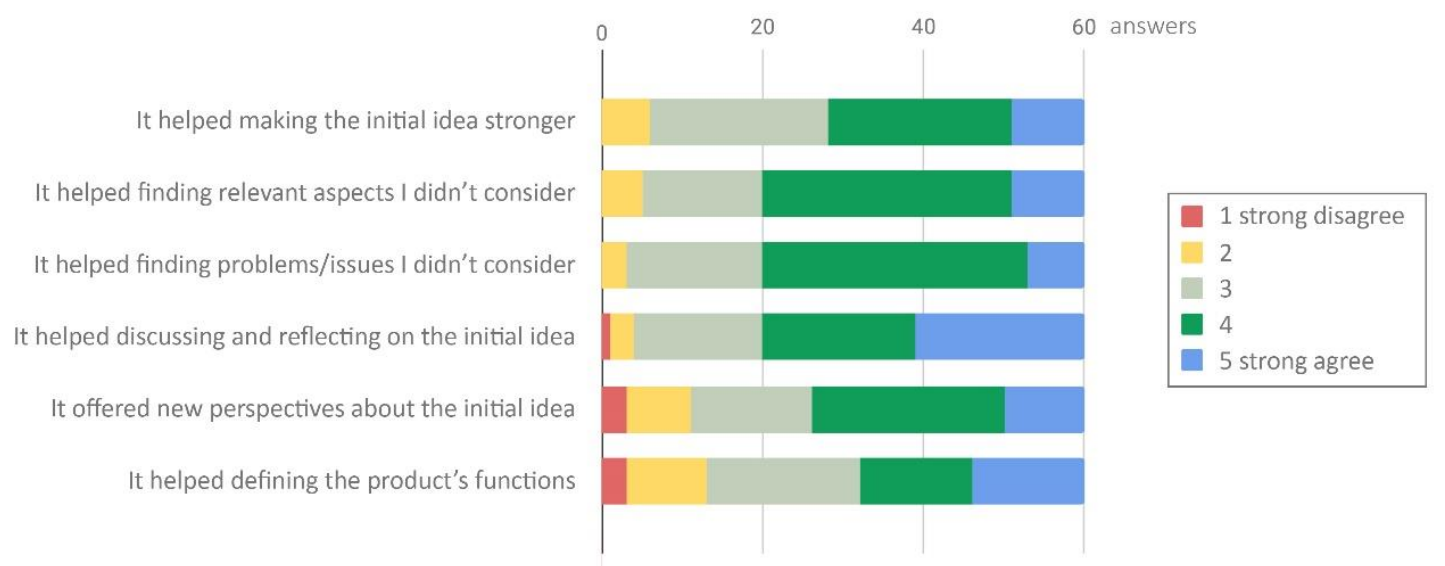

Figure 6: Summary of survey responses, evaluating the impact of the Mapping the loT Toolkit on the design project 
The MTIoT Toolkit was mostly appreciated as a support for discussing and reflecting on the concept with the design team (avg. rate 3.9). The tangible cards facilitated the development of personal visions that through discussion merged in a single common vision. They assisted in clarifying the purpose and direction of the product, while maintaining a ludic approach: for some participants, the whole exercise was seen as a game.

The critical perspective offered by the cards was aknowledged. The questions were a continuous push to deepen and inquire the concept. They were recognized as useful to identify new elements of the project, and to spot possible issues. "It helped finding relevant aspects I didn't consider" and "It helped finding problems and issues I didn't consider" were both rated 3.7 in average. "It is like a continuous review of the project", said one participant.

"Useful for group discussion. It let space for everyone to express their thoughts and allowed to think about aspects that we hadn't deepened - such as problems concerning technical-technological components and feasibility"

The MTloT Toolkit helped also students that weren't designing a technological product.

"We used it to improve our project, that wasn't a smart product, but still some cards were very useful for the discussion"

Questions were also appreciated as source of inspiration (avg. rate 3.6) during the brainstorming phase. "Questions can stimulate and enrich, steering the brainstorming" commented a partecipant.

The tangible aspect of the Toolkit was seen as a positive feature, liked by 30 participants out of 60 (50\%): 10 participants said that they would prefer a digital version, while 19 said that they would use both versions.

"The physical aspect is very important. Touching the cards and exchanging them through the group is very useful"

In some cases, the Toolkit was able to make the initial idea better/stronger (average rate 3.5 out of 5) and helped to define the product's functions (avg. 3,4). Overall the cards were considered useful (avg. 3.8), and the canvas too (avg. 3.7). Participants considered positive the methological aspect of the Toolkit and the exercises guiding the design process of smart products.

"I would use it again. I think it is a tool for students, professionals and for whoever wants to design following a guide during the different phases"

Although it was decided to test the toolkit with groups that already had a concept, the tool was considered relevant to be used during the initial phases of the design process, when there is more freedom of action on the project.

"It was very useful, I wish we had it presented before in the concept ideation phase" said a participant in test $A$. "We used it in an advanced stage of the project, we should have used it also before" said a participant of test $B$

Some negative aspects of the exercise with the Toolkit were:

- The lenght of the exercise: it can get quite long when a group deepens too many cards

- Some questions in the cards were perceived as repetitive

- The tool didn't help in defining the product's shape (average rate 2.3 out of 5), but it was useful to design the interaction.

\section{Conclusions}

To summarize: the change in user's lifestyles, new sociocultural models and technologies are drivers of meaning-driven innovation. They lead to the demand of different meanings, a divergent "why" for products to exist. This is particularly true for smart connected products, that are technological objects with a physical and digital component (hardware and software).

This paper outlines three different kind of meanings that are relevant in the design of smart connected products: 
1. The meaningful identity of the object as product category: smart products are not strongly culturally semantized. Designing a smart version of an existing product (analogic, or already electronic), or designing a new artifact that results in a new product on the market (e.g. the Roomba vacuum cleaner) requires an alignment with user's lifestyles. Connected products should have a strong identity that differentiates them from -or relates them to- the pre-existing meanings of their unconnected, "less smart" counterparts. Their role in the market needs to be clear, otherwise they will be labeled as smartphone accessories, "products with apps".

2. The meaning of the product in relation to its shape and functionality. For electronic products the link between shape and function is often lost: the functions can't be perceived, nor are geometrically comprehensible. Designers have the opportunity to reduce semantic infelicities by producing shapes that express the way of interaction, and that use metaphors to bring the meaning of the product closer to its primary function.

3. The meaning of the product in relation to a phygital ecosystem. The phygital nature of loT products blurs the boundaries between products and services, and leads to the need to design multiple touchpoints, both tangible and digital. IoT products should be social, in relationship with a compatible ecosystem of physical and virtual things, data, and processes. It is important to frame the role and meaning of the different phygital touchpoints and features, in relation to the user's expectations, while being aware of technical limitations.

The paper explored the notion that to achieve meaning-driven innovation is important to start from a strong vision coming from the inside-out of the company, rather than relying on HCD methods (Norman \& Verganti, 2014). A meaningful personal vision can be reached through critical thinking, discussion, and with a drive to deepen and challenge the design aspects of the product.

The tests with the "Mapping the loT" Toolkit saw that in action. Two classes of Master's degree students at the Design School of Politecnico di Milano were challenged in the design/redesign of smart products. They used the "sum up your smart-product idea" canvas and the "Mapping the loT card deck" in a group activity with their teams, to summarize and deepen their initial concepts.

The results emerging from the two described tests are:

1. It was appreciated to have a support for critical discussion with the team, as it helped to develop personal visions and to achieve a common one together.

2. The questions on the cards were a constant challenge for the initial idea. By answering and discussing, the concept was deepended, especially in terms of technical/technological aspects, and interaction.

3. Tangible cards were acknowledged as a good tool for teamwork, with a ludic approach.

4. Participants considered positive the methological aspect of the Toolkit and the exercises guiding the design process of smart products.

Therefore, the tests positively evaluated the use of question-cards to challenge the vision of the product and create a shared, more meaningful one.

The point is not that they miss ideas. they are overwhelmed by ideas. But they keep going in the same direction" (Verganti, 2007)

The Toolkit is published with a Commons License, and freely downloadable on its website (mappingtheiot.polimi.it). It wants to facilitate the design process of smart connected products, helping to design a meaningful product/service offer. It has been tested and presented in many occasions, both academically, and with companies and professionals.

Now, the aim is to make the "Mapping the loT" Toolkit grow: the team is open to collaboration to make it evolve. The discipline of product design can positively contribute to the area of smart products. Complex connected products require a renewed culture that takes elements from different design fields, such as product design, interaction design and service design, to generate new and coherent meaning ecosystems for positive and evolved user experiences. 


\section{References}

Adobe Blog (2017). UX Meets IoT: Designing Human Interactions with Smart Devices. Retrieved from https://theblog.adobe.com/ux-meets-iot-designing-human-interactions-smartdevices/?origref=https\%3A\%2F\%2Fwww.google.it\%2F

Apple (2018, 4 Dec 2018), Apple watch - Real stories. Retrieved from https://www.youtube.com/watch?v=0tqB4jnCxqA

Agrò, L. (2018). loT designer: Progettare oggetti e servizi relazionali. Franco Angeli Digitale.

Arquilla, V., \& Vitali, I. (2016). Designing in the loT Era: role and perspectives in design practices. In 6th International Forum of Design as a Process Systems \& Design: Beyond Processes and Thinking (pp. 871882). Editorial Univertitat Politècnica de València.

Battistella, C., Biotto, G., \& De Toni, A. F. (2012). From design driven innovation to meaning strategy. Management Decision, 50(4), 718-743.

Bower, J. L., \& Christensen, C. M. (1995). Disruptive technologies: catching the wave.

Breschi, R., Freundt, T., Orebäck, M., \& Vollhardt, K. (2018). The expanding role of design in creating an end-toend customer experience. McKinsey. Retrieved from https://www.mckinsey.com/businessfunctions/operations/our-insights/the-expanding-role-of-design-in-creating-an-end-to-end-customerexperience

Dahlin, K. B., \& Behrens, D. M. (2005). When is an invention really radical? Defining and measuring technological radicalness. research policy, 34(5), 717-737.

Diaz, J. (2019, 01 Jan 2019). The TV gets its first major redesign in decades. Fastcompany. Retrieved from www.fastcompany.com/90288998/the-tv-gets-its-first-major-redesign-indecades?partner=rss\&utm_source=facebook.com\&utm_medium=social\&utm_campaign=rss+fastcompany \&utm_content=rss\&fbclid=IwAR1K_4e_VzbIr6NWssfx2maJ4Xr9XVR2ERvwMzkJ6PV6KKMpJT8ILiqDPk4

Diller, S., Shedroff, N. and Rhea, D. 2005, Making Meaning: how successful businesses deliver meaningful customer experiences, New Riders Publishing, Berkeley, California, USA.

Friedman, R. (1986), Psychological Meaning of Products: Identification and Marketing Applications, Psychology and Marketing, 3 (Spring), 1-15

Friedmann, R. and Lessig, V.P. 1986, A Framework of Psychological Meaning of Products, in Lutz, R.J and Provo, UT (Eds.) 1986, North American Advances in Consumer Research, Volume 13, Association for Consumer Research, pp 338-34

Giaccardi, E. (2005). Metadesign as an Emergent Design Culture. Leonardo, 38(4), 342-49.

Giaccardi E., Fischer G. (2006). Meta-Design: A Framework for the Future of End User Development. in H. Lieberman, F. Paternò, \& V. Wulf (Eds.), End User Development, Kluwer Academic Publishers, Dordrecht, The Netherlands, pp. 427-457

Giacomin, J. (2017). What is Design for Meaning? Journal of design, business \& society, 3(2), 167-190.

Gibson. J. J. (1977). The Theory of Affordances, in R. Shaw and J. Bransford (2017) "Perceiving, Acting and Knowing: Toward an Ecological Psychology". Routledge.

Greenfield Adam (2006). Everyware: The dawning age of ubiquitous computing. New Riders Publishing, 2006

Google Design, Jitkoff, N., Design Is Never Done. Retrieved from https://design.google/library/design-neverdone/

Gutierrez, C., Garbajosa, J., Diaz, J., \& Yague, A. (2013). Providing a consensus definition for the term "Smart Product". In Proceedings of the International Symposium and Workshop on Engineering of Computer Based Systems, (December 2014), 203-211.

Hirschman, Elizabeth (1980), Attributes of Attributes and Layers of Meaning, in Advances in Consumer Research, Volume 7, Jerry Olson (ed), Ann Arbor, MI: Association for Consumer Research, 7-11 
INFSO D.4 Networked Enterprise \& RFID INFSO G.2 Micro \& Nanosystems, in Co-operation with the Working Group RFID of the ETP EPOSS, (2008) Internet of Things in 2020, Roadmap for the Future.

ITU. (2005). The internet of things. ITU Internet Reports - Executive Summary, 1-28. https://doi.org/10.1109/IEEESTD.2007.373646

Kazmierczak, E. T. (2003). Design as meaning making: from making things to the design of thinking. Design Issues, 19(2), 45-59.

Kim, W. C., Mauborgne R. (2005). Blue ocean strategy: from theory to practice. California management review, 47(3), 105-121.

Krippendorff, K. (1989). Product semantics: A triangulation and four design theories.

Krippendorff, K., \& Butter, R. (1984). Product Semantics Exploring the Symbolic Qualities of Form. Departmental Papers (ASC), 40.

Kiritsis, D. (2011). Closed-loop PLM for intelligent products in the era of the Internet of things. Computer-Aided Design, vol. 43, no. 5, pp. 479-501, May 2011

Maass W., Janzen S. (2007). Dynamic Product Interfaces: A Key Element for Ambient Shopping Environments. 20th Bled E-conference.

Maier, J. R., \& Fadel, G. M. (2009). Affordance based design: a relational theory for design. Research in Engineering Design, 20(1), 13-27.

Norman, D. A., \& Verganti, R. (2014). Incremental and Radical Innovation: Design Research vs. Technology and Meaning Change. Design Issues, 30(1), 78-96.

Rowland, C., Goodman, E., Charlier, M., Lui, A., \& Light, A. (2015). Designing Connected Products: UX for the Consumer Internet of Things. O'Reilly Media, Inc.

Sabou M., Kantorovitch J., Nikolov A., Tokmakoff A., Zhou X., Motta E., (2009). Position Paper on Realizing Smart Products: Challenges for Semantic Web Technologies. Networks, pp. 135-147.

Semmelhack P. (2013). Social Machines: How to Develop Connected Products That Change Customers' Lives. John Wiley \& Sons Ltd

Szalay, L. B., \& Deese, J. (1978). Subjective meaning and culture: An assessment through word associations. Lawrence Erlbaum Associates.

Ventä, O. (2007). Intelligent Products and Systems. VTT PUBLICATIONS, vol. 635.

Verganti, R. (2011). Radical design and technology epiphanies: A new focus for research on design management. Journal of Product Innovation Management, 28(3), 384-388.

Verganti, R. (2017). Overcrowded: designing meaningful products in a world awash with ideas. MIT Press.

Vitali (2015), Mapping the lot: un percorso di ricerca, analisi e sperimentazione in ambito Internet of Things. Master's Thesis. Politecnico di Milano

Vitali, I., Arquilla, V. (2018). Developing a Design Toolkit for the Internet of Things. In DRS 2018 International Conference (Vol. 3, pp. 1159-1174). Design Research Society.

Vitali, I., Rognoli, V., \& Arquilla, V. (2016, October). Mapping the IoT: Co-design, Test and Refine a Design Framework for loT Products. In Proceedings of the 9th Nordic Conference on Human-Computer Interaction (p. 142). ACM.

World Design Organization (2015). Definition of Industrial Design. Retrieved from http://wdo.org/about/definition/

You, H. C., \& Chen, K. (2007). Applications of affordance and semantics in product design. Design Studies, 28(1), 23-38. 